\title{
A Study on the Aberrant Methylation of Colorectal Cancer MLH1 Gene
}

\author{
Tingting Chen ${ }^{1,}$, Hongna Lv ${ }^{1}$, Yanping Xu ${ }^{1}$, Wenjing Du ${ }^{2}$, Rui Zhang ${ }^{3}$ \\ ${ }^{1}$ Department of Gastroenterology, Binzhou People's Hospital, Binzhou City, P. R. China \\ ${ }^{2}$ Technical Office, Binzhou Intermediate People's Court, Binzhou City, P. R. China \\ ${ }^{3}$ Technical Office, People's Court of Bincheng District, Binzhou City, P. R. China
}

Email address:

Chentingting2019@163.com (Tingting Chen)

*Corresponding author

\section{To cite this article:}

Tingting Chen, Hongna Lv, Yanping Xu, Wenjing Du, Rui Zhang. A Study on the Aberrant Methylation of Colorectal Cancer MLH1 Gene. American Journal of Clinical and Experimental Medicine. Vol. 7, No. 1, 2019, pp. 1-6. doi: 10.11648/j.ajcem.20190701.11

Received: January 17, 2019; Accepted: January 31, 2019; Published: February 1, 2019

\begin{abstract}
The aim of this paper is to study the mechanism of MLH1 gene expression and regulation in colorectal cancer, and to clarify the impact of 5' end CpG island aberrant methylation on the regulation of MLH1 gene expression. RT-PCR assay was used to detect the expression of MLH1 gene mRNA in 10 colon cancer cell strains. 62 fresh colorectal adenocarcinoma samples and paired distal normal mucosa were selected in our hospital. They comprised 37 males and 25 females aging 32 to 67 years old with the mean of 43.6. The cellular RNA was extracted, and immunohistochemistry assay was used to detect the expression of MLH1 protein in colorectal cancer, and MSP method was utilized to examine the methylation status of the first exon of MLH1 gene in the tissues. In the 10 cell strains, only the MLH1 mRNAs of SW480 and Caco2 were expressed, and no MLH1 mRNA expression was detected in the other eight strains. The expression level of MLH1 mRNA in the 62 cases of cancer tissue was higher than that in the paired normal mucosa, and MLH1 protein was mainly expressed in the cytoplasm. The expression level of MLH1 protein in adenocarcinoma tissues was significantly higher than that in the paired normal tissue $(\mathrm{P}<0.01)$, while the methylation level of cancer tissues was statistically significantly lower than that of the paired normal mucosa $(\mathrm{P}<0.05)$. Aberrant DNA methylation dominates the regulation of the MLH1 gene expression changes in colorectal cancer. The results herein provide a theoretical basis for clarifying the differential expressions of MLH1 in colorectal cancer in vitro and in vivo.
\end{abstract}

Keywords: Rectal Cancer, Colon Cancer, MLH1, Gene Expression and Regulation, Aberrant Methylation

\section{Introduction}

Colorectal cancer is a common digestive malignancy that endangers human health. Out of the fatal malignant tumors in the residents of the developed Western countries, colorectal cancer ranks second after lung cancer [1]. Over the past decade, the incidence of colorectal cancer is increasing particularly in urban and developed rural areas. For example, in urban areas of China, the incidence of colon cancer grows at a rate of $4.2 \%$ annually [2]. With China's socio-economic development and improvement of people's living standards, the dietary structure of residents has transited from the diet based on carbohydrates and high fibers (e.g. grain, vegetables) in the past to that based on high fat, high protein and low fiber (e.g. meat, eggs, milk), thus inducing colorectal cancer [3].
The results also indicate that in the 21 st century, the incidence of colorectal cancer will continue to rise in China.

The occurrence and development of colorectal cancer is a complex multi-gene process with multiple steps. With the in-depth research on tumor molecular biology, more genes have been found to be involved in the process of colorectal cancer occurrence, among which the activation of oncogenes and the inactivation of tumor suppressor genes are two important aspects of the genetic changes [4]. MLH1, as an important gene in the family of human mismatch repair genes (hMMR), plays a crucial role in maintaining the integrity and stability of the genetic information and in avoiding the generation of genetic mutations. MLH1 functional defects, which are considered to dominate tumor incidence, have been spotlighted in recent years [5]. Studies have shown that MLH1 is closely related to colorectal cancer, esophageal cancer, 
gastric cancer and cervical cancer, etc. [6]. However, there are few studies on the mechanism of expression and regulation of MLH1 gene in colorectal cancer. Therefore, the interpretation of this mechanism will help further clarify the effects of MLH1 on the occurrence and development of colorectal cancer, which is beneficial to the prevention and treatment of colorectal cancer.

\section{Materials and Methods}

\subsection{Materials}

All cancer tissues were fresh surgical specimens obtained from our hospital from March 2012 to February 2013, which were diagnosed as colorectal adenocarcinoma by pathological confirmation postoperatively. The samples were taken immediately after separation and then stored in liquid nitrogen. A total of 62 cases of colorectal adenocarcinoma and paired distal normal mucosa were selected. In 62 patients, there were 37 males and 25 females, with the mean age of 43.6 years old (32 to 67 years old).

Ten colon cancer cell strains of Hce8693, SW480, SW620, SW1116, HT29, RKO, HCT8, COLO205, Caco2 and CW2 were purchased from Beijing Ruixianghe Biotech Co., Ltd., and calf serum culture medium was obtained from Beijing ComWin Biotech Co., Ltd.

\subsection{Extraction of Total RNA in Cells}

All cells were cultured in $10 \%$ heat-inactivated calf serum medium in a saturated humidity incubator containing $5 \% \mathrm{CO}_{2}$ at $37^{\circ} \mathrm{C}$. The cells in exponential growth phase were collected, washed with PBS 3 times, added $1 \mathrm{ml}$ of TRIzol for repeated pipetting to be clear, and then placed at room temperature for $10 \mathrm{~min}$. After adding $0.3 \mathrm{ml}$ of chloroform, the solution was fully mixed, placed at room temperature for $30 \mathrm{~min}$, centrifuged at $4^{\circ} \mathrm{C}$ and $3,000 \mathrm{rpm} / \mathrm{min}$ for $10 \mathrm{~min}$, added $1 \mathrm{~m}$ of pre-cooled $60 \%$ ethanol to wash RNA precipitation after discarding the supernatant, and centrifuged at $3,000 \mathrm{rpm} / \mathrm{min}$ for $10 \mathrm{~min}$. Then the precipitation was blow-dried in super clean bench, added $50 \mu \mathrm{l}$ of $0.2 \%$ DEPC water, and then placed at $4{ }^{\circ} \mathrm{C}$ for $30 \mathrm{~min}$ until it was completely dissolved.

\subsection{Semi-quantitative RT-PCR}

First strand cDNA was synthesized using M-MLV reverse transcriptase with Oligo (dT) 16 as the primer, into which 5.0 $\mu \mathrm{g}$ total RNA was transcribed reversely. The upstream and downstream primers of MLH1 and G3PDH were designed respectively on different exons, and spaced by intron in the middle to avoid the interference of the product of genomic DNA amplification. Primer sequences of MLH1: upstream: 5'-AATTTCGGCCTGAACGGCAA-3', downstream: 5'-AAACCTCCTGGCTTGAAC-3'. Primer sequences of G3PDH: upstream: 5'-GGTCCCAATTTGCTTCAATT-3', downstream: 5'-CTTAAATGGCTTAAACGCCT-3'. PCR amplification conditions: long denaturation at $94^{\circ} \mathrm{C}$ for $2 \mathrm{~min}$, denaturation at $66^{\circ} \mathrm{C}$ for $30 \mathrm{~s}$, annealing at $56^{\circ} \mathrm{C}$ for $45 \mathrm{~s}$, extension at $72^{\circ} \mathrm{C}$ for $40 \mathrm{~s}$ for 40 cycles in total, and extension at $72^{\circ} \mathrm{C}$ for $3 \mathrm{~min}$ [7].

\subsection{Detection of MLH1 Protein in Colorectal Cancer by Immunohistochemical Method}

Adenocarcinoma and distal paired normal colorectal tissues were taken for paraffin embedding, then sliced at $3 \mu \mathrm{m}$ in thickness for each section, deparaffinized with dimethylbenzene three times (10 min each), washed with PBS three times (10 min each), added $3 \% \mathrm{H}_{2} \mathrm{O}_{2}$-methanol for 10 min to block endogenous peroxidase, microwave-repaired with pH 7.2 sodium citrate buffer (total power: $1200 \mathrm{~W}, 30$ $\mathrm{min})$, cooled in cold water at room temperature, washed with PBS three times (10 min each), blocked with 1:10 calf serum at room temperature for $30 \mathrm{~min}$, dropwise added MLH1 polyclonal antibody (1:100), placed in wet box, and incubated overnight at $4{ }^{\circ} \mathrm{C}$. The primary antibody was replaced with PBS as the negative control. The positive section was used as the positive control.

The expression level of MLH1 was graded from 0 to 3 according to the classification of Landberg et al.: Grade 0: no MLH1 expression in colorectal epithelial cells; Grade 1: weak positive expression; Grade 2: moderate expression; Grade 3: high expression. As MLH1 was uniformly expressed in tumor cells, the positive cells were not counted.

\subsection{Nested Methylation Specific PCR Amplification}

By the nested MSP assay, a pair of outer primers was used to amplify the large fragment containing targeted gene, and then the inner primer was used for amplification with the large fragment as a template to obtain the targeted band. The first cycle of PCR was used the outside primers, for which the reaction conditions were as follows: warm start at $92^{\circ} \mathrm{C}$ for 3 min, addition of $1.0 \mathrm{U}$ TaqDNA polymerase, thermal denaturation at $92^{\circ} \mathrm{C}$ for $60 \mathrm{~s}$, annealing at $56^{\circ} \mathrm{C}$ for $40 \mathrm{~s}$, extension at $72^{\circ} \mathrm{C}$ for $30 \mathrm{~s}$ for 30 cycles in total, and extension at $60^{\circ} \mathrm{C}$ for $1 \mathrm{~min}$. The product of the first cycle PCR was diluted to be $1: 100,1 \mu 1$ of which was taken as a template for the second cycle. The annealing temperature was increased to $72^{\circ} \mathrm{C}$, while the other conditions of the reaction were the same as those of the first cycle. The PCR reaction system comprised $10 \times$ PCR buffer $(3.0 \mu \mathrm{l}), 5 \mathrm{mM}$ dNTP $(3.0 \mu \mathrm{l}), 10 \mu \mathrm{M}$ upstream and downstream primers $(1 \mu \mathrm{l}$ each), and template $(1 \mu \mathrm{l})$. Methylated DNA was taken as the positive control of methylation, and unmethylated DNA as the positive control of unmethylation. The primer sequences used in the MSP amplification were as follows: outer-S: CCGGGTAATTCTACGTTCGAACTTGT, outer-AS: TCAACAATTTCCCCAAAAGGCTCCTCCAAC, MSP (M)-S: TAGCAAAGCCGACGCT, MSP (M)-AS: AGCGCTACCTCCGAACG, and MSP (U)-S: CCAAACCTATAACCGT, MSP (U)-AS: AAACTTTGGGACTCCCT [8].

\subsection{Statistical Analysis}

All data were analyzed by SPSS13.0, and median and interquartile range (P25 P75) were used to describe the 
central tendency and dispersion trend of the data. Since all cancer and normal tissues were paired, paired rank sum test (two-sample Wilcoxon method) was adopted to analyze the differences in MLH1 expression and methylation in colorectal cancer and paired normal colorectal tissues. $\mathrm{X}^{2}$ test or Fisher exact probability was used to analyze the relationship between the methylation status and each clinicopathological parameter, $\mathrm{P}<0.05$ was considered as significantly different.

\section{Results}

\subsection{Expression of MLH1 mRNA in Colon Cancer Cell Strains}

Semi-quantitative RT-PCR assay was used to detect the expression of MLH1 gene mRNA in 10 colon cancer cell strains. In the 10 cell strains, only MLH1 mRNAs of SW480 and Caco2 were expressed, and no MLH1 mRNA expression was detected in the other eight cell strains (Figure 1).

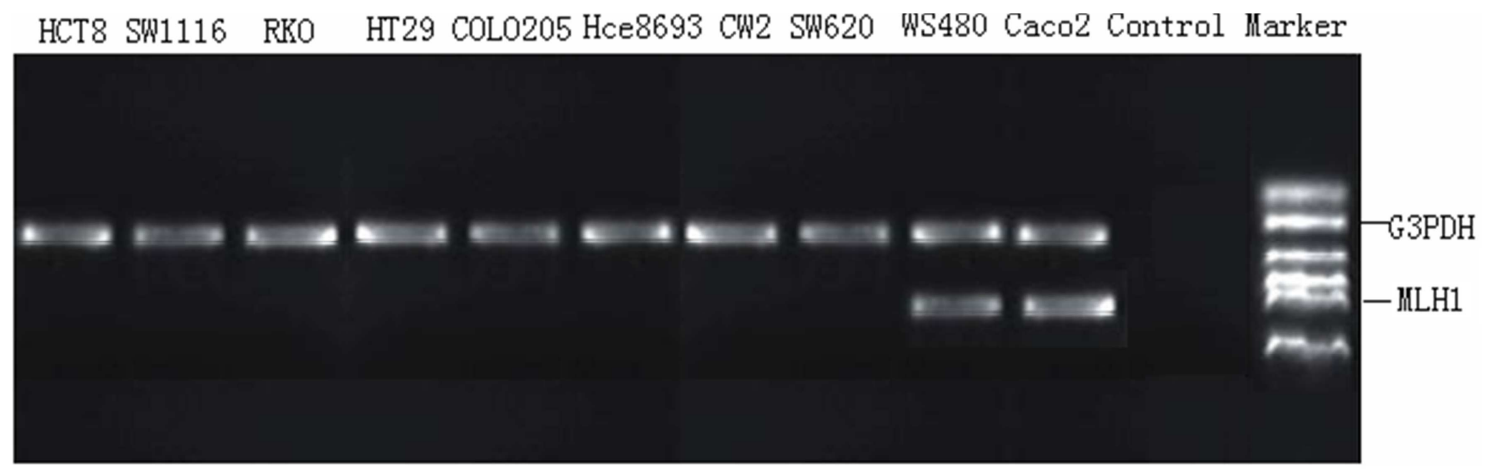

Figure 1. Expression of MLH1 mRNA in colon cancer cell strains

\subsection{Expression of MLH1 in Colorectal Cancer Tissues}

On the mRNA level, the relative expression level of MLH1 in the 62 cases of cancer tissues was higher than that in the paired normal mucosa. The MLH1 expressions in some colorectal cancer and paired normal tissues are shown in Figure 2. By paired rank sum test, the expression level of MLH1 in 62 cases of colorectal cancer tissues was significantly higher than that in the paired normal mucosa $(\mathrm{P}<0.01)$ (Table 1). Immunohistochemistry results show that MLH1 protein was expressed mainly in the cytoplasm. The expression level of MLH1 protein in adenocarcinoma tissues was significantly higher than that in the paired normal tissue $(\mathrm{P}<0.01)$ (Table 2, Figure 3, Figure 4A, 4B).

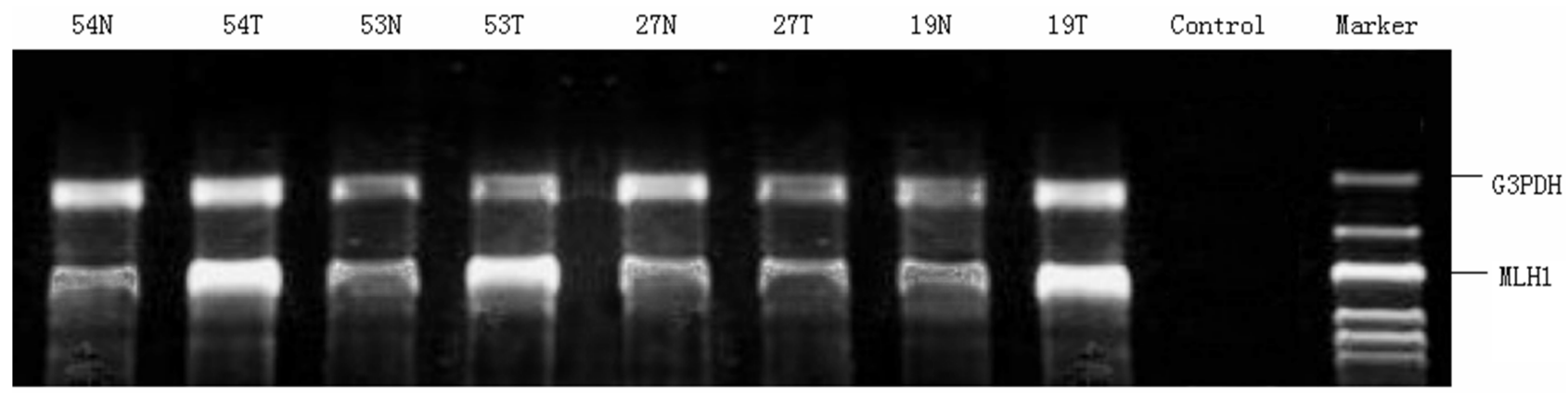

Figure 2. MLH1 mRNA expressions in some colorectal cancer and paired normal tissues. N: normal tissue; T: cancer tissue. Numbers represent case No.

Table 1. Relative expression levels of MLH1 gene in 62 cases of colorectal cancer tissues.

\begin{tabular}{lllll}
\hline & Case No. & Median & P25 & P75 \\
\hline Normal tissue & 62 & 0.19 & 0.05 & 0.42 \\
Cancer tissue & 62 & 0.57 & 0.26 & 0.879 \\
\hline
\end{tabular}

Relative expression of MLH1 = MLH1 pixels/G3PDH pixels P25 and P75 represent interquartile range values.

Table 2. Expressions of MLH1 protein in colorectal cancer and paired normal tissues.

\begin{tabular}{lllllll}
\hline & \multicolumn{9}{c}{ MLH1 expression } & \\
\hline & Case No. & $\mathbf{0}$ & $\mathbf{1}$ & $\mathbf{2}$ & $\mathbf{3}$ & P \\
\hline Normal tissue & 62 & 15 & 34 & 11 & 2 & $<0.01$ \\
Cancer tissue & 62 & 9 & 29 & 17 & 7 & \\
\hline
\end{tabular}




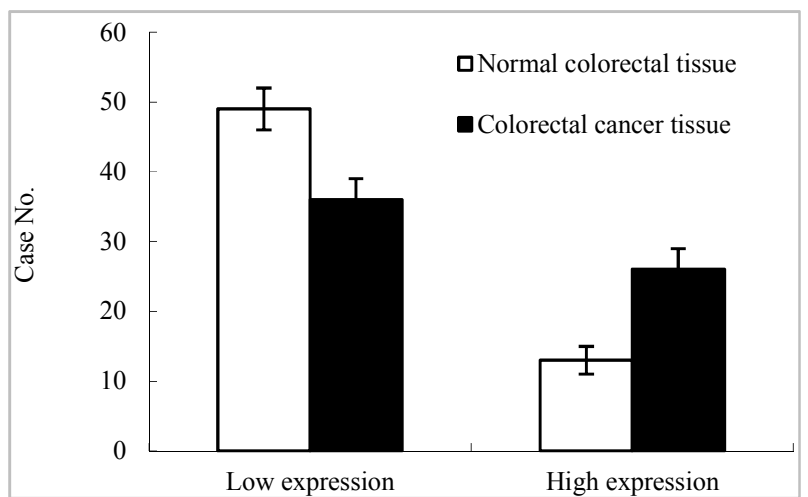

Figure 3. Expressions of $M L H 1$ protein in colorectal cancer and paired normal tissues. Low expression, Grade 0 and 1; high expression, Grade 2 and 3
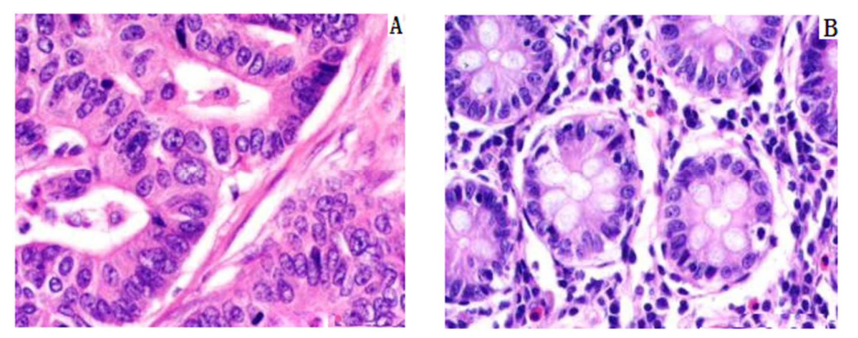

Figure 4. Expressions of MLH1 protein in colorectal cancer tissues and paired normal mucosa. A, normal colorectal tissue; B, colorectal cancer tissue.

\subsection{Detection of MLH1 Gene Methylation in First Exon by MSP Method}

MSP assay was used to detect the methylation status of MLH1 gene in the first exon CpG island. MLH1 methylation was observed in 39 cases $(39 / 62,62.90 \%)$ of colorectal cancer tissues (including full methylation and partial methylation), while in paired normal mucosa, 51 cases $(51 / 62,82.25 \%)$ experienced methylation. The methylation level of cancer tissues was lower than that of the paired normal mucosa, between which the difference was statistically significant $(\mathrm{P}<0.05)$ (Table 3, Figure 5).

Table 3. Methylation status of MLH1 in 62 cases of paired tissues.

\begin{tabular}{lllll}
\hline & Case No. & Methylation & Unmethylation & P \\
\hline Normal tissue & 62 & 51 & 11 & $<0.05$ \\
Cancer tissue & 62 & 39 & 23 & \\
\hline
\end{tabular}

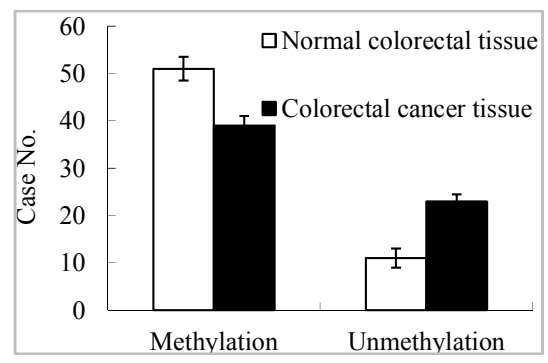

Figure 5. Methylation status of MLH1 in colorectal cancer tissues and paired normal mucosa.

\subsection{Correlation Between MLH1 Methylation in First Exon and Clinical Pathological Data}

The results of $\mathrm{X}^{2}$ test and Fisher exact probability show that the methylation of tumor tissues was not correlated with the age, gender, tumor location, degree of differentiation and other clinicopathological parameters of patients $(\mathrm{P}>0.05)$ (Table 4).

Table 4. Correlation between MLH1 methylation in first exon and clinical pathological data.

\begin{tabular}{|c|c|c|c|c|}
\hline & \multirow{2}{*}{ Case No. } & \multicolumn{2}{|c|}{ Methylation status } & \multirow{2}{*}{$\mathbf{P}$} \\
\hline & & Unmethylation & Methylation & \\
\hline Age & & & & 0.627 \\
\hline$<60$ & 30 & 11 & 19 & \\
\hline Gender & & & & 0.154 \\
\hline Male & 37 & 14 & 23 & \\
\hline Female & 25 & 9 & 16 & \\
\hline Colon & 32 & 10 & 22 & \\
\hline Rectum & 30 & 13 & 17 & \\
\hline Differentiation degree & & & & 0.106 \\
\hline High differentiation & 24 & 9 & 15 & \\
\hline Low differentiation & 38 & 14 & 24 & \\
\hline
\end{tabular}

\section{Discussions}

Tumorigenesis is a complex process with multiple gene changes and multi-event accumulation, including the activation of a series of oncogenes and inactivation of tumor suppressor genes. It is currently believed that there are mainly two mechanisms that cause abnormal gene expression. One is the genetic mechanism, i.e. the abnormal gene expression caused by changes of nucleotide sequences of DNA, such as nucleotide mutations or the increase or decrease of gene copy number. The other is the epigenetic mechanism, referring to changes of ways of genetic gene expression that does not involve changes in DNA sequences $[9,10]$. DNA methylation is a mode of action the most thoroughly studied. Most mammalian DNA methylations occur on cytosine in $\mathrm{CpG}$ dinucleotide. CpG-rich DNA sequence known as $\mathrm{CpG}$ island, is mainly located in the promoter and the first exon region of a gene [11]. Except the $x$ chromosome inactivation and the $\mathrm{CpG}$ island of imprinted gene, the autosomal $\mathrm{CpG}$ islands usually do not appear methylation. In case of $\mathrm{CpG}$ island methylation, 
gene transcription is inhibited [12].

The overall hypomethylation of tumor genome and hypermethylation of regulatory sequences of some genes (specific tumor suppressor genes) is one of the characteristics of tumor molecular changes. The role of gene hypermethylation in tumorigenesis has been a concern to scholars in the past two decades, and there are a lot of literature reports [13]. Inactivated genes for hypermethylation are involved in almost all of the signaling pathways in the occurrence and development process of cancer, such as DNA repair genes (hMLH1, MGMT, BRCA1), cell cycle regulatory genes (p16, p14, p15), apoptosis-related genes (DAPK, APAFl), carcinogen metabolism-related gene (GSTPl), hormonal response gene (RARB2) and cell adhesion molecules (CDH1, CDH3), etc. [14, 15]. A lot of evidences have indicated that the methylation of $\mathrm{CpG}$ islands in gene promoter and the first exon region can inhibit the expression of specific genes [16].

DNA hypomethylation is widespread in genomes, and overall genome hypomethylation is also one of the reasons for tumorigenesis. A large number of studies have found that hypomethylation of the genome as a whole exists in a variety of human tumors [17]. It is now believed that there are two mechanisms for the effect of hypomethylation in tumors, which is as follows: first, hypomethylation/demethylation can activate some proto-oncogenes; second, hypomethylation makes chromatin and genome instable and mutation frequency increased. However, it has been poorly understood so far about the specific effect of hypomethylation in 5' end promoter of specific gene and the first exon region on the tumorigenesis [18, 19]. It has been found that some genes show hypomethylation in tumor tissues but hypermethylation in paired normal tissues, and thereafter it is also found that hypomethylation of the c-fos and c-myc genes is associated with increased expression of the corresponding genes [20]. But it is until recent years that the research on hypomethylation and increased expression of some tumor genes has received attention gradually, and it is also found that hypomethylation of multiple genes is related to tumorigenesis [21].

Tumor cells are living in the stromal microenvironment of the host, so the changes of this microenvironment may exert a great influence on many steps of tumor progression, such as tumor cell invasion and metastasis, which is mainly achieved by the interaction between tumor cells and their surrounding stromal cells (such as fibroblasts, endothelial cells or immune cells) [22]. In normal tissues, a dynamic balance is maintained between epithelial cells and their surrounding microenvironment (fibroblasts, endothelial cells, immune cells and extracellular matrix, etc.). And in the process of malignant transformation and progress, there is also a interaction between tumor cells and their surrounding stromal cells. Stromal changes can promote tumor progression [23]. Therefore, in colorectal cancer tissues, the changes of MLH1 gene expression and the demethylation of its first exon $\mathrm{CpG}$ island are likely to be regulated by growth factor signaling pathways, chemical factors and cell adhesion molecules in the microenvironment [24]. Regardless, these assumptions also need the evidence of further experimental researches.

\section{Conclusion}

In summary, MLH1 gene has the presence of aberrant methylation in colorectal cancer tissues. Increased MLH1 expression in colorectal cancer tissues is related to the hypomethylation of its first exon $\mathrm{CpG}$ island. This study again confirms that aberrant DNA methylation is an important regulatory mechanism for changes of MLH1 gene expression in colorectal cancer.

\section{References}

[1] Heitman SJ. Hilsden RJ. et al. Correction: Colorectal Cancer Screening for Average-Risk North Americans: An Economic Evaluation [J]. PLoS Med, 2017, 9 (11): 648-52.

[2] Dai Z. Zheng RS. et al. Analysis and prediction of colorectal cancer incidence trend in China [J]. Zhonghua Yu Fang Yi Xue Za Zhi, 2017, 46 (7): 598-603.

[3] Wan DS. Epidemiological trend and control strategy of colorectal cancer in China [J]. Zhonghua Zhong Liu Za Zhi, 2018, 33 (7): 481-3.

[4] Gonzalez-Gonzalez M. Garcia JG. et al. Genomics and proteomics approaches for biomarker discovery in sporadic colorectal cancer with metastasis [J]. Cancer Genomics Proteomics, 2017, 10 (1): 19-25.

[5] Martinez-Uruena N. Alvarez LM. et al. Incidence of -93 MLH1 promoter polymorphism in familialand sporadic colorectal cancer [J]. Colorectal Dis, 2016, 31 (2): 226-9.

[6] Wang T. Liu Y. et al. Association between MLH1 -93G>a polymorphism and risk of colorectal cancer $[\mathrm{J}]$. PLoS One, 2017, 7 (11): 449-51.

[7] Rouleau E. Lefol C. et al. Quantitative PCR high-resolution melting (qPCR-HRM) curve analysis, a new approach to simultaneously screen point mutations and large rearrangements: application to MLH1 germline mutations in Lynch syndrome [J]. Hum Mutat, 2017, 30 (6): 867-75.

[8] Zhang Z. Sun D. et al. Development of a non-invasive method, multiplex methylation specific PCR (MMSP), for early diagnosis of nasopharyngeal carcinoma [J]. PLoS One, 2017, 7 (11): 908-10.

[9] Tsujii M. Cyclooxygenase, cancer stem cells and DNA methylation play important roles in colorectal carcinogenesis [J]. Digestion, 2017, 87 (1):12-6.

[10] Lange CP. Campan M. et al. Genome-scale discovery of DNA-methylation biomarkers for blood-based detection of colorectal cancer [J]. PLoS One, 2016, 7 (11): 266-8.

[11] Gao Y. Killian K. et al. Leukocyte DNA methylation and colorectal cancer among male smokers [J]. World J Gastrointest Oncol, 2016, 4 (8): 193-201.

[12] Fang WJ. Zheng Y. et al. Genome-wide analysis of aberrant DNA methylation for identification of potential biomarkers in colorectal cancer patients [J]. Asian Pac J Cancer Prev, 2017, 13 (5): 1917-21. 
[13] Williams EA. Folate, colorectal cancer and the involvement of DNA methylation [J]. Proc Nutr Soc, 2017, 71 (4): 592-7.

[14] Bosch LJ. Mongera S. et al. Analytical sensitivity and stability of DNA methylation testing in stool samples for colorectal cancer detection [J]. Cell Oncol (Dordr), 2018, 35 (4): 309-15.

[15] Li BQ. Yu H. et al. MicroRNA Mediated Network and DNA Methylation in Colorectal Cancer [J]. Protein Pept Lett, 2017, $62(8): 556-8$.

[16] Xicola RM. Llor X. et al. DNA methylation defects in sporadic and hereditary colorectal cancer [J]. Gastroenterol Hepatol, 2017, 35 (7): 480-7.

[17] Shen Y. Takahashi M. et al. Boswellic acid induces epigenetic alterations by modulating DNA methylation in colorectal cancer cells [J]. Cancer Biol Ther, 2018, 13 (7): 542-52.

[18] Zhao ZW. Lian WJ. et al. Decreased expression of repulsive guidance molecule member A by DNA methylation in colorectal cancer is related to tumor progression [J]. Oncol Rep, 2016, 27 (5): 1653-9.

[19] Cassinotti E. Melson J. et al. DNA methylation patterns in blood of patients with colorectal cancer and adenomatous colorectal polyps [J]. Int J Cancer, 2017, 131 (5): 1153-7.

[20] Hinoue T. Weisenberger DJ. et al. Genome-scale analysis of aberrant DNA methylation in colorectal cancer [J]. Genome Res, 2018, 22 (2): 271-82.

[21] Kaneda A. Yagi K. et al. Two groups of DNA methylation markers to classify colorectal cancer into three epigenotypes [J]. Cancer Sci, 2017, 102 (1): 18-24.

[22] Kim JW. Park HM. et al. Polymorphisms in genes involved in folate metabolism and plasma DNA methylation in colorectal cancer patients [J]. Oncol Rep, 2018, 25 (1): 167-72.

[23] Ward RL. Dobbins T. et al. Identification of constitutional MLH1 epimutations and promoter variants in colorectal cancer patients from the Colon Cancer Family Registry [J]. Genet Med, 2016, 15 (1): 25-35.

[24] Nakanishi R. Kitao H. et al. FANCJ expression predicts the response to 5-fluorouracil-based chemotherapy in MLH1-proficient colorectal cancer [J]. Ann Surg Oncol, 2017, 19 (11): 3627-35. 